\title{
Clinical features in osteosarcoma and prognostic implications
}

\author{
Ângela Rech ${ }^{1}$, Cláudio G. Castro Jr. ${ }^{2}$, Jane Mattei ${ }^{3}$, Lauro Gregianin ${ }^{4}$, \\ Luciane Di Leone ${ }^{5}$, Alexandre David6, Luis F. Rivero7, Ricardo Tarrago8, \\ Armando Abreu' ${ }^{9}$, Algemir L. Brunetto ${ }^{10}$
}

\begin{abstract}
Objective: To identify the clinical features in osteosarcoma and to investigate their influence on the prognosis of children and adolescents presenting this disease.

Material and methods: The records of children and adolescents with osteosarcoma treated by the Bone Tumors Group of the state of Rio Grande do Sul, Brazil, between January 1992 and December 2001 were reviewed.

Results: Fifty consecutive patients were included in this study. Mean age at diagnosis was 13 years $(3-22) ; 68 \%$ of the patients were males. The primary site of disease was the femur in $50 \%$ of the patients, tibia in $30 \%$, pelvis in $4 \%$, humerus in $10 \%$, fibula in $2 \%$ and other sites in $4 \%$. Nineteen patients presented metastases at diagnosis $(38 \%)$. All patients received chemotherapy and were treated with three different schemes. As for surgical treatment, 26 patients $(52 \%)$ had an amputation and $17(34 \%)$ received conservative surgery. Serum lactic dehydrogenase > $1,000 \mathrm{UI} / \mathrm{ml}(\mathrm{p}=0.0159, \log$ rank), tumor necrosis $<90 \%$ and presence of metastases had a negative influence on prognosis. The overall 5 -year survival was of $33.2 \pm 7.2 \%$ with mean follow-up of 36 months (6-126). Event-free survival was $29.7 \pm 7 \%$. The 5 -year event-free survival in non-metastatic patients was $45 \pm 10.7 \%$, and zero in metastatic patients (follow-up of 78.4 and 18.7 months, respectively). Only two out of 19 metastatic patients are alive and free of disease at 18 and 30 months respectively.

Conclusion: Metastatic disease at diagnosis, serum levels of serum lactic dehydrogenase $>1,000 \mathrm{UI} / \mathrm{ml}$ and tumor necrosis $<90 \%$ are predictors of unfavorable prognosis. The excessively high incidence of metastatic patients may suggest the presence of an aggressive pattern of disease in our population, or may indicate late diagnosis.
\end{abstract}

J Pediatr (Rio J). 2004;80(1):65-70: Osteosarcoma, metastatic, children.

1. Resident physician, Service of Pediatric Oncology, Hospital de Clínicas de Porto Alegre (HCPA), Porto Alegre, RS, Brazil.

2. MSc. Universidade Federal do Rio Grande do Sul (UFRGS). Pediatric Oncologist, Service of Pediatric Oncology, HCPA, Porto Alegre, RS, Brazil.

3. Medical student, School of Medicine, Universidade Federal do Rio Grande do Sul (UFRGS), Porto Alegre, RS, Brazil.

4. PhD. Pediatric Oncologist, Service of Pediatric Oncology, HCPA, Porto Alegre, RS, Brazil.

5. MSc. Nurse, Chief of the Department of Clinical Assays, Service of Pediatric Oncology, HCPA, Porto Alegre, RS, Brazil.

6. Orthopedist. Chief of the Grupo de Tumores do Aparelho Locomotor, Santa Casa de Misericórdia, Porto Alegre, RS, Brazil.

7. Professor, School of Medicine, UFRGS. Pathologist, Pathology Service, HCPA, Porto Alegre, RS, Brazil.

8. Pathologist. Chief of the Unit of Bone Tumors, Santa Casa de Misericórdia and Fundação Faculdade de Ciências Médicas de Porto Alegre (FFCMPA), Porto Alegre, RS, Brazil.

9. Radiologist, Hospital Mãe de Deus, Porto Alegre, RS, Brazil.

10. PhD. Chief of the Pediatric Oncology Service, HCPA. Associate professor, School of Medicine, UFRGS. Professor, School of Medicine, Universidade Luterana do Brasil, Canoas, RS, Brazil.

Manuscript received Feb 25 2003, accepted for publication Sep 172003.

\section{Introduction}

Osteosarcoma (OS) is a primary malignant bone tumor that is more frequent in children, adolescents and young adults and whose peak incidence occurs in the second decade of life, accounting for approximately $5 \%$ of malignant diseases among children and adolescents. 1,2

In the last 25 years, there has been a remarkable improvement in the prognosis of OS patients, especially in those with localized disease. Before the 1970s, these patients were treated only with surgery, but more than $50 \%$ of them showed systemic recurrence of the disease in less than six months and $90 \%$ of them died due to the progression of the disease. After 1970, several randomized studies showed that the combination of chemotherapy and surgery significantly improved the cure rates. ${ }^{1}$ In the last two decades, the quality of orthopedic prostheses has been remarkably improved and conservative surgeries 
have been used more frequently; these improvements have contributed to the better quality of life of these patients. ${ }^{3}$

Patients with localized disease have a better prognosis than those with metastatic disease. Age, volume, site, and resectability of the primary tumor, as well as serum LDH level and response to neoadjuvant chemotherapy are clinical characteristics with known prognostic importance. This way, patients with a favorable prognosis may benefit from less aggressive treatments, whereas the role of therapeutic programs that use cancer drugs with a higher dose intensity or clinical trials with new drugs has been investigated for patients at a higher risk of recurrence.

Knowing the clinical characteristics of OS patients in our setting allows for the identification of important strategies for the development of therapeutic protocols adapted to the Brazilian reality.

\section{Materials and methods}

The authors reviewed the medical records of 50 consecutive patients diagnosed with OS between January 1992 and December 2001. All patients were treated by an interdisciplinary team, following the routines for diagnosis and treatment discussed in a weekly meeting throughout the study period. The inclusion criteria were: patients with histological diagnosis of high-grade OS, ${ }^{4}$ age less than 22 years, and treatment compliance. This retrospective study was approved by the Research and Ethics Committee of Hospital de Clínicas de Porto Alegre.

The evaluation sheet included the following data: gender, race, city of origin, birthdate, histological diagnosis, site of the primary tumor and of the metastatic lesions, symptoms at presentation, serum level of lactic dehydrogenase (LDH) at presentation. Information about clinical status, type of surgery, and tumor necrosis factor was also collected.

Three consecutive treatment protocols, approved by the Research and Ethics Committee of Hospital de Clínicas de Porto Alegre (HCPA), were used during the study period. The therapeutic strategies used in these protocols consisted of chemotherapy before and after resection of the primary tumor and of the metastatic lesions (if present). The major objectives of providing presurgical chemotherapy are to assess pathological response, to favor conservative surgical treatment and to allow time for the manufacture of prosthetic devices.

The following treatment protocols were used: European Osteosarcoma Intergroup (EOI), which consisted of six cycles of cisplatin and doxorubicin, between 1992 and 1995; Brazilian Osteosarcoma Protocol - Study IV, with cycles of cisplatin, carboplatin and doxorubicin, between 1996 and 1999; and the Brazilian Protocol for Metastatic and Non-Metastatic Osteosarcoma, in 2000, with cisplatin, ifosfamide, and doxorubin, totaling nine cycles and two more cycles of cyclophosphamide for patients with metastases at diagnosis, after 2000.

The statistical data were assessed using Epi-Info, including also the analysis of frequency variables and survival curve (Kaplan-Meir method), and univariate analysis of the major risk factors.

\section{Results}

The medical records of 50 consecutive patients diagnosed with high-grade os were reviewed. The age range was 3 to 22 years (median of 13.5 years), with 34 male patients $(68 \%)$ and 16 female patients $(32 \%) ; 48$ were white $(96 \%)$ and two were black $(4 \%)$.

Histological types were as follows: conventional: 41 patients $(82 \%)$; teleangiectatic: seven patients $(14 \%)$; small cells: one patient ( $2 \%)$. This information could not be retrieved from the medical records of one patient $(2 \%)$.

The sites of the tumor were: femur: 25 (50\%); tibia: 15 (30\%); iliac bone: two (4\%); humerus: five (10\%); fibula: one (2\%). This information was lost in two cases $(4 \%)$.

Of 19 patients (38\%) with metastatic disease at diagnosis, 17 had pulmonary metastases, one had liver metastases and one had bone marrow metastases.

As far as symptoms at diagnosis are concerned, 23 cases were related to trauma $(46 \%)$ and 21 showed pathological fracture $(42 \%)$. Sixty-two per cent of the patients had difficulty in walking; other symptoms included: pain $(78 \%)$, increase in volume $(50 \%)$, edema $(38 \%)$, heat or calor $(10 \%)$ and redness or rubor $(8 \%)$.

The following treatment protocols were used: European Osteosarcoma Intergroup (66\%); Brazilian Osteosarcoma Protocol - Study IV (12\%); Brazilian Protocol for Metastatic and Non-Metastatic Osteosarcoma (16\%), and others $(6 \%)$.

With regard to surgical treatment, 26 (52\%) patients were submitted to amputation; 17 (34\%) underwent conservative surgery, among whom two received autologous bone graft and arthrodesis, two were submitted to conservative surgery with irradiated bone graft and arthrodesis, one had extra-articular resection and placement of an endoprosthesis, one had resection of the tumor and vascularized fibular graft, one underwent resection of the tumor, fibular graft and plate, and 10 received a conventional prosthesis. Of the remaining patients, three refused to be operated on, and four contained no information on their medical records.

Overall survival was $33.2 \pm 7.2 \%$ with an average follow-up of 36.76 months $(6-126)$ and disease-free survival was $29.7 \pm 7 \%$ (Figure 1 ).

The tumor necrosis factor was assessed in 27 patients $(54 \%)$, and ranged from 5 to $100 \%$ (mean $=72 \%$; standard deviation of $29.6 \%$ ). Of 12 patients with a tumor necrosis factor equal to or greater than $90 \%$, the overall 
survival was $61.4 \pm 15.3 \%$; of 15 patients with a tumor necrosis factor less than $90 \%$, the overall survival was $35.7 \pm 12.8 \%(R R=2.71 \mathrm{p}=0.011$ and log rank $=0.0535)$ (Figure 2).

The probability of disease-free survival at five years of patients with nonmetastatic tumors at diagnosis was $45 \pm 10.7 \%$ and zero for metastatic tumors (mean follow up of 78.4 and 18.7 months, respectively) (Figure 3); of 19 patients with metastatic disease, only two are not on treatment and free of disease with a follow up of 18 and 30 months, respectively.

The probability of overall survival at five years was $33.2 \pm 7.2 \%$ with a mean follow-up of 36.8 months (6-126) and disease-free survival was $29.7 \pm 7 \%$ (Figure 1).

Serum LDH levels were obtained at diagnosis in 44 patients (88\%). Thirty eight patients with LDH levels lower than $1,000 \mathrm{IU} / \mathrm{ml}$ had an overall survival of $32.9 \% \pm 8.4 \%$, whereas in six patients with LDH greater than $1,000 \mathrm{IU} / \mathrm{ml}$ the overall survival was $16.7 \% \pm 15.2 \%$ (log rank = 0.0159).

After the treatment, 15 patients (30\%) showed tumor recurrence. The tumor recurred in the lung of 10 patients. The treatment of the recurrent tumor consisted of chemotherapy in five patients (34\%), and chemotherapy and surgery in eight patients (54\%). Two patients did not accept treatment

\section{Discussion}

The profile of the patients described in the present study is not different from that observed in other studies.
Most patients showed involvement of long bones, especially of the femur and tibia, and predominance of conventional histology. The presence of initial symptoms related to trauma and/or pathological fracture in at least $25 \%$ of the patients should serve as a warning to pediatricians, who should consider the hypothesis of neoplasm in children and adolescents with persistent trauma-related osseous pain, and have a radiological investigation performed as soon as possible. Suspicious bone lesions should be biopsied, preferably by a surgeon with experience in bone tumors. Once the histological diagnosis of OS is confirmed, the extension of the disease has to be determined by means of staging tests. The treatment of os requires resection with tumor-free margins and use of systemic chemotherapy, ${ }^{5}$ even if the primary tumor has been totally resected, because many patients have microscopic metastatic disease. 6

Three chemotherapy protocols were used during the study period, with three distinct periods of inclusion in different multi-institutional studies.

Some randomized studies have suggested that there exists no difference in disease-free survival between immediate surgery followed by adjuvant chemotherapy and presurgical chemotherapy. ${ }^{7}$ However, most studies recommend that the patient be submitted to surgery only after being treated with chemotherapy. This approach has allowed an increasing number of patients who respond well to chemotherapy to benefit from conservative surgery. In our study, a significant number of patients underwent amputation, which results from the detection of a large tumor at diagnosis, and has a possible negative effect on survival; ${ }^{3}$ those patients who could be treated with

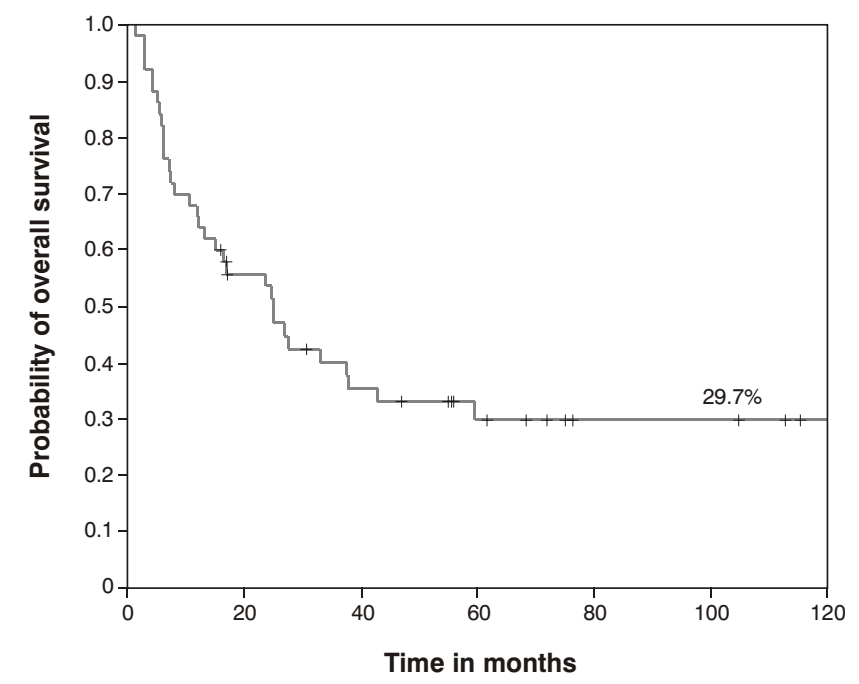

Figure 1 - Disease-free survival curve. 


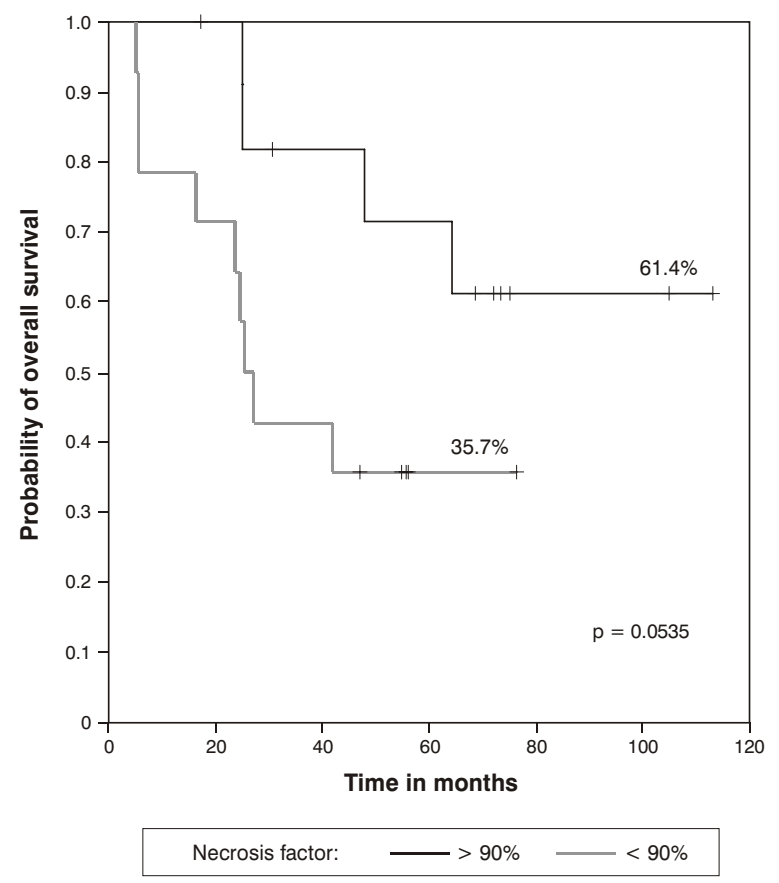

Figure 2 - Overall survival curve according to the tumor necrosis factor.

conservative surgery benefited from bone grafts and conventional prostheses, including three patients who received irradiated autologous bone graft and arthrodesis, as described in a recent case report. ${ }^{8}$ It is necessary to highlight the importance of a careful evaluation of candidates for conservative surgery, especially those with large tumors. This decision should be preferably made by an interdisciplinary team with experience in the management of bone tumors.

Unfavorable prognostic factors for osteosarcoma include the presence of metastatic disease at diagnosis, ${ }^{9}$ resection of tumor with compromised margins, ${ }^{10}$ poor response to chemotherapy, ${ }^{10}$ large tumors ${ }^{11}$ and elevated serum levels of LDH. ${ }^{12}$ Male patients aged less than 10 years with elevated serum levels of alkaline phosphatase also have been suggested as having a poor prognosis. ${ }^{10}$ Quite recently, certain molecular characteristics, such as the presence of multiple-drug resistant gene, 13 loss of heterozygosity on the RB gene $\mathrm{P}^{13}$ and increased expression of HER2/erbB-2 genes, 14,15 seem to be unfavorable to prognosis.

The presence of metastatic disease has been regarded as the worst prognostic factor in both univariate and multivariate analyses; fewer than $20 \%$ of these patients have a disease-free survival at five years. ${ }^{10}$ The less favorable results in these patients are probably related to the fact that they have a large tumor with less possibility of total resection than to the fact that they are more resistant to chemotherapy. ${ }^{10}$ The interval between the onset of symptoms and diagnosis is shorter in patients with metastatic disease than in patients with localized disease, probably translating into a more aggressive behavior of the disease in the former ones. ${ }^{11}$ Among patients with metastatic disease, those with pulmonary lesions tend to have a better response to the treatment; however, patients with bone or bone marrow metastases have an extremely limited prognosis. ${ }^{10}$ Of 19 patients in our study with metastatic disease at diagnosis, only two are still alive, with no signs of the disease. Therefore, in the present study, the presence of metastasis at diagnosis was invariably associated with recurrence, regardless of the metastatic site.

We used serum LDH levels to know the extension of the disease. ${ }^{12}$ In the Multi-Institutional Osteosarcoma Study, ${ }^{16}$ elevated LDH level at diagnosis was related to higher treatment failure and adverse prognostic factor for patients with nonmetastatic os of the extremities submitted to adjuvant chemotherapy. ${ }^{1}$ The univariate analysis of our patients revealed that only those patients with a very high $\mathrm{LDH}$ level ( $>1,000 \mathrm{IU} / \mathrm{ml}$ ) were at greater risks for recurrence. A larger sample would allow us to observe the influence of intermediate levels (above 2 standard deviations within the upper control limit) of LDH over the prognosis. 


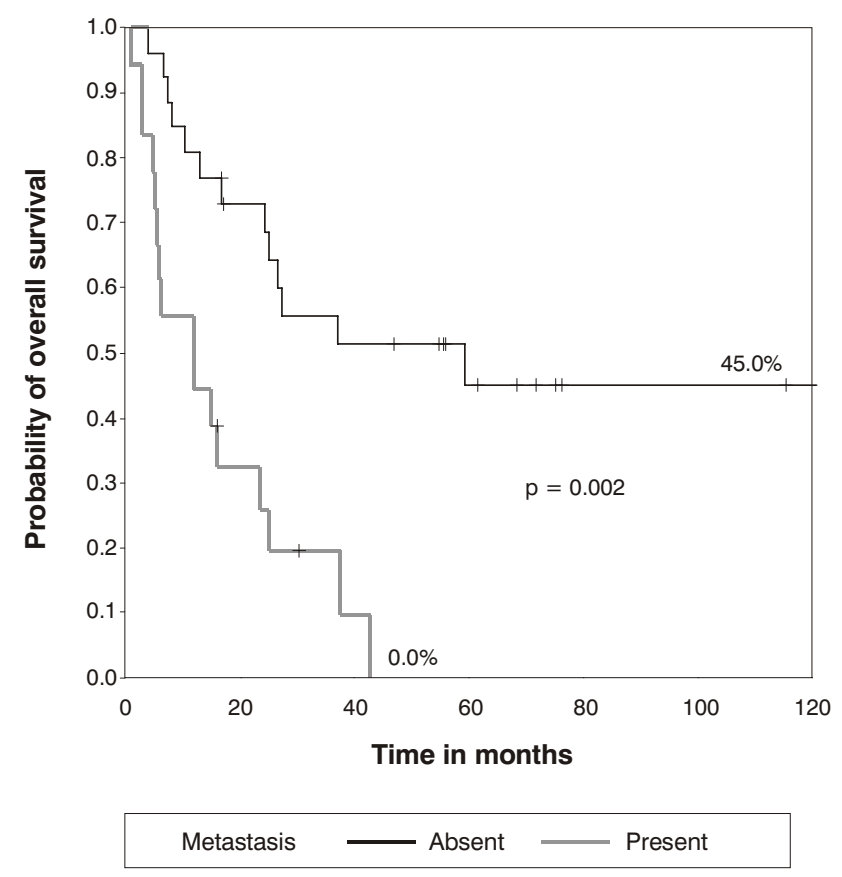

Figure 3 - Disease-free survival curve of patients with metastatic tumors and nonmetastatic tumors.

Several studies have consistently demonstrated that tumor response to presurgical chemotherapy is an important indicator of longer survival for patients with localized disease; ${ }^{17}$ the level of response may be assessed by determining the tumor necrosis factor by way of magnetic resonance, ${ }^{17}$ by the histological determination of the tumor necrosis factor in the resected specimen or through clinical parameters. ${ }^{18-20}$ However, most studies have used the histological grading of the tumor as a criterion for tumor response. The assessment of the histological response was made in only $54 \%$ of the patients, but it allowed us to confirm the prognostic importance of this variable.

In conclusion, the presence of metastatic disease, serum LDH levels greater than 1,000 IU/ml at diagnosis, or tumor necrosis factor less than $90 \%$ at presurgical chemotherapy are poor prognostic factors. The excessively large number of patients with metastatic disease at diagnosis suggests that these patients have an aggressive type of osteosarcoma, or else they are diagnosed too late in our setting. We should attempt to establish the diagnosis of OS as soon as possible. The treatment of these patients in Brazilian studies is crucial for us to know the clinical, epidemiological and biological characteristics of this type of tumor, allowing us to improve treatment strategies.

\section{References}

1. Link M, Eilber F. Osteosarcoma. In: Pizzo P, Poplack D. Principles and Practice of Pediatric Oncology. 4th ed. Philadelphia: Lippincott - Raven Publishers; 2002. p. 1051-1089.

2. Gurney JG, Swensen AR, Bulterys M. Bone Tumor. In: Cancer Incidence and Survival among Children and Adolescents: United States SEER Program 1975. 1999;994649:99-109.

3. Petrilli AS, Gentil FC, Epelman S, Lopes LF, Bianchi A, Lopes A, et al. Increased survival, limb preservation, and prognostic factors for osteosarcoma. Cancer. 1991;68:733-7.

4. Schajowicz F, Ackerman LV, Sisson HA. Histological typing of bone tumors. International Histological Classification of Tumors. N.6. Geneva; 1992.

5. Meyers P, Gorlick R. Osteossarcoma. In: Link M. Clínicas Pediátricas da América do Norte. Belo Horizonte: Interlivros; 1997. p. 981-999.

6. Bieling $P$, Rehan $N$, Winkler $P$. Tumor size and prognosis in aggressively treated osteosarcoma. J Clin Oncol. 1996;14: 848-58.

7. Goorin A, Baker A, Giese P. No evidence for improved event free survival with presurgical chemotherapy for non-metastatic extremity osteogenic sarcoma: preliminary results of randomized Pediatric Oncology Group trial 8651 [abstract]. Proceedings of the American Society of Clinical Oncology 1995;14:A-1420. n. 444.

8. Davi A, Rios AR, Tarrago R, Rotta NW, Brunetto AL, Abreu A. Auto-enxerto tumoral irradiado nas reconstruções dos tumores ósseos. Revista Brasileira de Ortopedia. 2000;35:325-32.

9. Meyer WH, Pratt CB, Poquette CA, Rao BN, Parham DM, Marina NM, et al. Carboplatin/Ifosfamide Window Therapy for Osteosarcoma: Results of the St Jude Children's Research Hospital OS-91 Trial. J Clin Oncol. 2001;19:171-82. 
10. Bielack SS, Kempf-Bielack B, Delling G, Exner GU, Flege S, Helmke $K$, et al. Prognostic factors in high-grade osteosarcoma of the extremities or trunk: an analysis of 1,702 patients treated on neoadjuvant cooperative osteosarcoma study group protocols. J Clin Oncol. 2002;20:776-90.

11. Bacci C, Ferrari S, Longhi A, Forni C, Zavatta M, Versari M, et al. High-grade Osteosarcoma of extremity: differences between localized and metastatic tumors at presentation. J Pediatr Hematol Oncol. 2002;24:27-30.

12. Pochanugool L, Subhadharaphandou $T$, Dhanachai M, Hathirat P, Sangthawan D, Pirabul R, et al. Prognostic factors among 130 patients with osteosarcoma. Clin Orthop. 1997;345:206-14.

13. Feugeas O, Guriec N, Babin-Boilletot A. Loss of heterozygosity of the RB gene is a poor prognostic factor in patients with Osteosarcoma. J Clin Oncol. 1996;14:467-72.

14. Gorlick R, Huvos AG, Heller G. Expression of HERZ/erb-2 correlates with survival in Osteosarcoma. J Clin Oncol. 1999; 17:2781-8.

15. Onda M, Matsuda S, Higalci S. ErbB-2 expression is correlated with poor prognosis for patients with osteosarcoma. Cancer. 1996;77(1):71-8.

16. Link MP, Goorin AM, Horowitz M. Adjuvant chemotherapy of high-grade osteosarcoma of the extremity. Updated results of the Multi-Institucional Osteosarcoma Study. Clin Orthop. $1991 ; 8-14$
17. Reddick WE, Wang S, Xiong X. Dynamic magnetic resonance imaging of regional contrast access as an additional prognostic factor in pediatric osteosarcoma. Cancer. 2001;91:2230-7.

18. Davis AM, Bell RS, Goodwin PJ. Prognostic factors in osteosarcoma: a critical review. J Clin Oncol. 1994;12:423-31.

19. Provisor AJ, Ettinger LJ, Nachman JB, Krailo MD, Makley JT, Huvos AG, et al. Treatment of non-metastatic osteosarcoma of the extremity with preoperative and postoperative chemotherapy: a report from the Children's Cancer Group. J Clin Oncol. 1997; 15:76-84.

20. Weeden S, Grimer RJ, Cannon SR. European Osteosarcoma Interproup: The effect of local recurrence on survival in resected osteosarcoma. Eur J Cancer. 2001;37:39-46.

Corresponding author:

Algemir Lunardi Brunetto

Hospital de Clínicas de Porto Alegre

Rua Ramiro Barcelos, 2350, $3^{\circ}$ andar leste

CEP 90035-007 - Porto Alegre, RS, Brazil

Tel./fax: +55 (51) 3330.8087

E-mail: abrunetto@hcpa.ufrgs.br 